J. Austral. Math. Soc. (Series A) 45 (1988), 66-77

\title{
PRIMITIVE PERMUTATION GROUPS WITH A DOUBLY TRANSITIVE SUBCONSTITUENT
}

\author{
CHERYL E. PRAEGER
}

(Received 5 June 1986)

Communicated by $\mathbf{H}$. Lausch

Dedicated to Robert Edwards in recognition of 25 years' distinguished contribution to mathematics in Australia, on the occasion of his retirement

\begin{abstract}
Let $G$ be a primitive permutation group on a finite set $\Omega$. We investigate the subconstitutents of $G$, that is the permutation groups induced by a point stabilizer on its orbits in $\Omega$, in the cases where $G$ has a diagonal action or a product action on $\Omega$. In particular we show in these cases that no subconstituent is doubly transitive. Thus if $G$ has a doubly transitive subconstituent we show that $G$ has a unique minimal normal subgroup $N$ and either $N$ is a nonabelian simple group or $N$ acts regularly on $\Omega$ : we investigate further the case where $N$ is regular on $\Omega$.
\end{abstract}

1980 Mathematics subject classification (Amer. Math. Soc.): 20 B 15, 05 C 25.

Finite primitive permutation groups with a doubly transitive subconstituent were first studied by W. A. Manning (see $[12,17.7]$ ). His results were generalized by P. J. Cameron ([2] and see also [4, 8, 9]). The analogues of these groups in the area of symmetric graphs, namely, 2-arc transitive graphs, have also received a great deal of attention in the literature. In this paper we show that these groups have a unique minimal normal subgroup which either is a nonabelian simple group or is regular. We begin by studying the nature of the subconstituents of a primitive group $G$ with a diagonal or a product action: we show in particular

The work for this paper was supported by a special CTEC grant at the University of Western Australia.

(C) 1988 Australian Mathematical Society 0263-6115/88 \$A2.00 + 0.00 
that no subconstituent is doubly transitive for these groups. Using the O'Nan Scott Theorem (see $[1,7$, or 11]) we can thén deduce immediately

THEOREM A. Let $G$ be a primitive permutation group on a finite set $\Omega$ such that the stabilizer of $\alpha \in \Omega$ is doubly transitive on one of its orbits in $\Omega \backslash\{\alpha\}$. Then either

(a) $T \leq G \leq$ Aut $T$ for a nonabelian simple group $T$, or

(b) $G$ has a unique minimal normal subgroup which is regular on $\Omega$.

We continue the investigation of those groups with a regular normal subgroup.

\section{Primitive groups with a simple diagonal action}

Here we assume that $G \leq X=N\left(\right.$ Aut $\left.T \times S_{k}\right)$ is a primitive permutation group on $\Omega$ where we assume the following.

(i) The group $N$ is the socle of $G$ and $N=T_{1} \times \cdots \times T_{k}$ is a direct product of $k \geq 2$ groups $T_{i}$, each isomorphic to a fixed nonabelian simple group $T$.

(ii) The group Aut $T$ acts naturally on each factor $T_{i}$ of $N$ and each inner automorphism $\sigma_{t}: x \rightarrow x^{t}$, for $x, t \in T$, is identified with a diagonal element $(t, t, \ldots, t)$ of $N$, that is Aut $T \cap N=D=\{(t, t, \ldots, t) \mid t \in T\}$.

(iii) The group $S_{k}$ permutes the set $\left\{T_{1}, \ldots, T_{k}\right\}$ naturally and the subgroup $P$ of $S_{k}$ induced by $G$ is either primitive of degree $k$, or $k=2$ and $P=1$.

(iv) We may assume that for some $\alpha \in \Omega, X_{\alpha}=$ Aut $T \times S_{k}$ with the group, of inner automorphisms of $T$ identified with the subgroup $D$ of $N$; then $D \leq$ $G_{\alpha} \leq A(T) \times P$ where $A(T)$ is the projection of $G_{\alpha}$ to Aut $T$ and $D \leq A(T)$. The points of $\Omega$ can be identified with the set of right cosets of $D$ in $N$ so that

$$
\alpha=D(1,1, \ldots, 1)
$$

and for $\beta=D\left(t_{1}, \ldots, t_{k}\right) \in \Omega, \mathbf{s}=\left(s_{1}, \ldots, s_{k}\right) \in N, \sigma \in \operatorname{Aut} T$ and $\tau \in S_{k}$ we have

$$
\begin{aligned}
& \beta^{\boldsymbol{\theta}}=D\left(t_{1} s_{1}, \ldots, t_{k} s_{k}\right), \\
& \beta^{\sigma}=D\left(t_{1}^{\sigma}, \ldots, t_{k}^{\sigma}\right), \\
& \beta^{\tau}=D\left(t_{1 \tau^{-1}}, \ldots, t_{k \tau^{-1}}\right) .
\end{aligned}
$$

Here we are taking $\tau$ to act on the set $\{1,2, \ldots, k\}$ in the natural way. We note that $|\Omega|=|T|^{k-1}$, and that each coset of $D$ has a unique member with $k$ th entry equal to 1 . Let $\Pi_{A}, \Pi_{P}$ denote the projections of $G_{\alpha}$ onto $A(T)$ and $P$ respectively. We shall investigate $G_{\alpha}$-orbits $\Delta$ which are quasiprimitive (that is all normal subgroups of $G_{\alpha}$ either fix $\Delta$ pointwise or are transitive on $\Delta$ ). We begin by showing that the only fixed point of $D$ in $\Omega$ is $\alpha$. 
LEMMA 1.1. The diagonal subgroup $D$ fixes only the point $\alpha=D(1, \ldots, 1)$.

ProOF. Suppose that $D$ fixes $\delta=D\left(t_{1}, \ldots, t_{k-1}, 1\right) \neq \alpha$. Since $\delta \neq \alpha$ at least one entry say $t_{i}$ is not the identity element of $T$. Now for $\mathbf{t}=(t, \ldots, t) \in D$ we have $\delta=\delta^{t}=D\left(t^{t}, t^{t}, \ldots, t_{k-1}^{t}, 1\right)$ and as each $D$-coset contains a unique element with $k$ th entry 1 we have $t_{i}=t_{i}^{t}$ : since this is true for all $t \in T$ it follows that $t_{i}$ is in the centre of $T$. This contradiction completes the proof.

Thus if $G_{\alpha}$ is quasiprimitive on one of its orbits $\Delta$ then $D$ is transitive on $\Delta$ :

LEMMA 1.2. Suppose that $D$ is transitive on a $G_{\alpha}$-orbit $\Delta \neq\{\alpha\}$, and that $\delta=D\left(t_{1}, t_{2}, \ldots, t_{k}\right) \in \Delta$. Then $t_{1}, t_{2}, \ldots, t_{k}$ are distinct elements of $T$.

ProOF. This is true if $k=2$ so suppose that $k \geq 3$ : then $P$ is primitive on $\left\{T_{1}, \ldots, T_{k}\right\}$. Suppose that exactly $x$ entries $t_{i}$ in $\left(t_{1}, t_{2}, \ldots, t_{k}\right)$ are equal to $t_{k}$; then $1 \leq x<k$ since $\delta \neq \alpha$. Let $i$ be an index for which $t_{i} \neq t_{k}$. Then for some $\tau \in P$ we have $i^{\tau}=k$, and for some $\sigma \in A(T)$ we have $\sigma \tau \in G_{\alpha}$ : thus $\Delta$ contains $\delta^{\sigma \tau}=D\left(t_{1 \tau^{-1}}^{\sigma}, \ldots, t_{k \tau^{-1}}^{\sigma}\right)$ with $k$ th entry $t_{k \tau^{-1}}^{\sigma}=t_{i}^{\sigma}$. However as $D$ is transitive on $\Delta, \delta^{\sigma \tau}=\delta^{\mathbf{t}}$ for some $\mathbf{t}=(t, \ldots, t) \in D$. It follows that exactly $x$ entries in $\left(t_{1 \tau^{-1}}^{\sigma}, \ldots, t_{k \tau^{-1}}^{\sigma}\right)$ are equal to $t_{k \tau^{-1}}^{\sigma}=t_{i}^{\sigma}$ and hence that exactly $x$ entries in $\left(t_{1}, \ldots, t_{k}\right)$ are equal to $t_{i}$. Thus the partition of $\{1,2, \ldots, k\}$ determined by equality of the elements $t_{1}, t_{2}, \ldots, t_{k}$ has $k / x$ blocks of size $x$. Further this partition is independent of the coset representative $\left(t_{1}, \ldots, t_{k}\right)$ chosen for $\delta$, and as $D$ is transitive on $\Delta$ this partition is independent of the point $\delta$ chosen from $\Delta$. Thus this partition must be preserved by $P$. Since $P$ is primitive and $x<k$ we must have $x=1$.

Next we investigate the case where $G_{\alpha}$ is unfaithful on $\Delta$.

LEMMA 1.3. Suppose that $\Delta \neq\{\alpha\}$ is a $G_{\alpha}$-orbit such that $D^{\Delta}$ is transitive and the kernel $K$ of $G_{\alpha}$ on $\Delta$ is nontrivial. The following hold.

(a) The group $K \leq G_{\alpha} \cap P$ and $K$ is regular on $\tau=\left\{T_{1}, \ldots, T_{k}\right\}$. Moreover if $\delta=D\left(t_{1}, t_{2}, \ldots, t_{k}\right) \in \Delta$ with $t_{k}=1$ then $S=\left\{t_{1}, t_{2}, \ldots, t_{k}=1\right\}$ is a subgroup of $T$ isomorphic to $K$ : the map $\varphi: K \rightarrow S$ defined by $\varphi(\tau)=t_{k \tau^{-1}}$ for $\tau \in K$ is an isomorphism.

(b) If $K=G_{\alpha} \cap P$ then $K \simeq S$ is elementary abelian, and $P$ is soluble. Further if $G_{\alpha}^{\Delta}$ is primitive then $G_{\alpha \delta}=G_{\alpha} \cap\left(N_{A(T)}(S) \times P\right)$ and $N(S) \cap\left(G_{\alpha} \cap A(T)\right)=$ $C(S) \cap\left(G_{\alpha} \cap A(T)\right)$ : in particular $S$ is not a Sylow subgroup of $G_{\alpha} \cap A(T)$. The orbit $\Delta$ is self-paired if and only if some element of $T$ inverts (each element of) $S$, and if $\Delta$ is self-paired and primitive then $S$ is an elementary abelian 2-group.

(c) If $G_{\alpha} \cap P$ is transitive on $\Delta$ then $G_{\alpha}^{\Delta}$ is primitive with two regular normal subgroups $\left(G_{\alpha} \cap P\right)^{\Delta}$ and $\left(G_{\alpha} \cap A(T)\right)^{\Delta}$ each isomorphic to $T$ (so $G_{\alpha} \cap A(T)=$ $D$ ). Moreover $S=T \simeq K$ and $G_{\alpha} \cap P \simeq T \times T$ so that $k=|T|$. (We can 
change the subscripts $1,2, \ldots, k$ of the $T_{i}$ to elements of $T$ and choose a coset representative for $\delta$ with $x$-entry $t_{x}$ and $t_{1}=1$ so that $K$ identified with $T$ acts on the subscripts by left multiplication. Then for some $\sigma=\sigma(\delta) \in \operatorname{Aut} T, t_{x}=x^{\sigma}$ for all $x \in T$ : and the stabilizer of $\delta$ in $\left(D \times\left(G_{\alpha} \cap P\right)\right)^{\Delta}$ is $\left\{\left(\mathbf{x}, \sigma x \sigma^{-1}\right) \mid x \in\right.$ $T\} \simeq T$, where $\mathbf{x}=(x, x, \ldots, x)$.)

REMARKS 1.4. (a) In part (a), $K$ is a regular normal subgroup of a primitive group $P$ and hence is elementary abelian or a direct power of a nonabelian simple group. We show in (b) that $K$ is elementary abelian when $G_{\alpha} \cap P=K$.

(b) If $G_{\alpha}^{\Delta}$ is quasiprimitive then $\left(G_{\alpha} \cap P\right)^{\Delta}$ is transitive or trivial: the latter case corresponds to $K$ being elementary abelian. In the former case we show in (c) that $K \simeq T$ and $G_{\alpha}^{\Delta}$ is in fact primitive with two regular normal subgroups isomorphic to $T$.

(c) If $G_{\alpha}^{\Delta}$ were 2-transitive then $G_{\alpha}^{\Delta}$ would have a unique minimal normal subgroup, namely $D^{\Delta}$, so case (c) could not hold. Further in none of the almost simple 2-transitive groups does $D_{\delta}^{\Delta}$ have a nontrivial centre, and hence case (b) does not hold either. Thus in Lemma 1.3, $G_{\alpha}^{\Delta}$ is not 2-transitive.

ProoF. (a) Since $D$ is faithful on $\Delta, K \cap A(T)=K \cap D=1$ and it follows that $K$ centralizes $D$. Hence $\Pi_{A}(K)$ centralizes $D$, and as $D$ has trivial centralizer in $A(T)$ we conclude that $\Pi_{A}(K)=1$, that is $K \subseteq P$. Thus we have $K \leq G_{\alpha} \cap P$, (so $P \neq 1$ ).

Now $K$ is normal in $\Pi_{P}\left(G_{\alpha}\right)=P$ and as $P$ is primitive on $\tau=\left\{T_{1}, \ldots, T_{k}\right\}$ it follows that $K$ is transitive on $\tau$. If $\tau \in K$ fixes $k$ then since $\delta=\delta^{\tau}=$ $D\left(t_{1 \tau^{-1}}, \ldots, t_{k \tau^{-1}}\right)$ with $t_{k \tau^{-1}}=t_{k}=1$ we must have $t_{i \tau^{-1}}=t_{i}$ for all $i$ : since all the $t_{i}$ are distinct it follows that $\tau=1$. Thus $K$ is regular on $\tau$.

Now for each $i \in\{1, \ldots, k\}$ there is a unique $\tau=\tau_{i} \in K$ such that $i^{\tau}=k$ : then $\delta^{\tau}=\delta$ yields $t_{j \tau^{-1}}=t_{i} t_{j}$ for each $j \in\{1, \ldots, k\}$. Thus $S=\left\{t_{1}, t_{2}, \ldots, t_{k}=1\right\}$ is closed under multiplication and hence is a subgroup of $T$. Further $\tau=\tau_{i}$ induces a permutation of $S$ by $t_{j}^{\tau}=t_{j \tau^{-1}}$ and we have seen that this permutation is simply left multiplication by $t_{i}$. Moreover by definition of $\tau_{i}$ we have $\tau_{i} \tau_{j}=\tau_{x}$ where $x=j \tau_{i}^{-1}$ and we also have $t_{i} t_{j}=t_{j \tau_{i}^{-1}}=t_{x}$. It follows that the map $\varphi\left(\tau_{i}\right)=t_{i}$, is an isomorphism.

(b) Assume next that $G_{\alpha} \cap P=K$. Then $A(T)=\Pi_{A}\left(G_{\alpha}\right) \simeq G_{\alpha} /\left(G_{\alpha} \cap P\right)=$ $G_{\alpha} / K, P / K=\Pi_{P}\left(G_{\alpha}\right) / \Pi_{P}(K) \simeq G_{\alpha} / K\left(G_{\alpha} \cap A(T)\right)$ and $K\left(G_{\alpha} \cap A(T)\right) / K \simeq$ $\left(G_{\alpha} \cap A(T)\right) /\left(K \cap\left(G_{\alpha} \cap A(T)\right)\right) \simeq G_{\alpha} \cap A(T)$. It follows that $P / K$ is isomorphic to a section of Out $T$ and hence $P / K$ is soluble. From [1] the only primitive groups $P$ with a regular normal subgroup $K$ such that $P / K$ is soluble are those of affine type, that is those with $K$ elementary abelian. 
Now $\Delta$ is self-paired if and only if $\Delta$ contains $\delta^{-1}=D\left(t_{1}^{-1}, t_{2}^{-1}, \ldots, t_{k}^{-1}\right)$, and as $D$ is transitive on $\Delta$, this is true if and only if some $t \in T$ inverts each of the $t_{i}$.

For $\gamma=D\left(s_{1}, s_{2}, \ldots, s_{k}\right) \in \Delta$, with $s_{k}=1$, let $S(\gamma)=\left\{s_{1}, s_{2}, \ldots, s_{k}=1\right\}$. Then as $D^{\Delta}$ is transitive $S(\gamma)$ is a subgroup of $T$ conjugate to $S$. The subset $\Delta_{S}=\{\gamma \in \Delta \mid S(\gamma)=S\}$ is clearly a block of imprimitivity for $G_{\alpha}$ in $\Delta$, and $\Delta_{S}$ contains $\delta^{-1}$ if $\Delta$ is self-paired. If $G_{\alpha}^{\Delta}$ is primitive then $\Delta_{S}=\{\delta\}$ (so that $\delta=\delta^{-1}$, that is $S$ is a 2-group, whenever $\Delta$ is self-paired). Now an element $\sigma \tau \in G_{\alpha}$, where $\sigma \in A(T), \tau \in P$, fixes $\Delta_{S}$ setwise if and only if $\sigma \in N_{A(T)}(S)$, that is the setwise stabilizer of $\Delta_{S}$ in $G_{\alpha}$ is $G_{\alpha} \cap\left(N_{A(T)}(S) \times P\right)$. Thus if $G_{\alpha}^{\Delta}$ is primitive then $G_{\alpha \delta}$ is $G_{\alpha} \cap\left(N_{A(T)}(S) \times P\right)$; moreover $\sigma \in G_{\alpha} \cap N_{A(T)}(S)$ fixes $\delta$ if and only if $\sigma$ centralizes $S$, so $G_{\alpha} \cap N_{A(T)}(S)=G_{\alpha} \cap C_{A(T)}(S)$ : by [6, 7.4.3] $S$ is not a Sylow subgroup of $G_{\alpha} \cap A(T)$.

(c) Finally assume that $G_{\alpha} \cap P$ is transitive on $\Delta$. Then $G_{\alpha}^{\Delta}=G_{\alpha} / K$ has normal subgroups $\left(G_{\alpha} \cap A(T)\right)^{\Delta}=\left(\left(G_{\alpha} \cap A(T)\right) \times K\right) / K$ and $\left(G_{\alpha} \cap P\right)^{\Delta}=$ $\left(G_{\alpha} \cap P\right) / K$ with trivial intersection. Thus $\left(G_{\alpha} \cap A(T)\right)^{\Delta}$ centralizes $\left(G_{\alpha} \cap P\right)^{\Delta}$ and as both are transitive it follows that both are regular and are isomorphic to each other: thus as $D^{\Delta}$ is transitive we have $G_{\alpha} \cap A(T)=D \simeq\left(G_{\alpha} \cap P\right) / K$. Now the stabilizer of $\delta$ in $D^{\Delta} \times\left(G_{\alpha} \cap P\right)^{\Delta}$ is a diagonal subgroup and hence for each $\mathbf{t}=(t, t, \ldots, t) \in D$ there is a $\tau \in G_{\alpha} \cap P$ such that $\delta^{\mathbf{t} \tau}=\delta$. If we define $S(\gamma)$ as above for $\gamma \in \Delta$, we have $S\left(\delta^{t \tau}\right)=S^{t}$ and hence $t$ normalizes $S$. Since this holds for all $t \in T$ we must have $S=T$. It follows that $G_{\alpha} \cap P=K \times L$ where $L \simeq\left(G_{\alpha} \cap P\right)^{\Delta} \simeq T$. Since $K$ is regular on $T$ we may replace the set of labels $\{1,2, \ldots, k\}$ by the set $T$ so that $K$ identified with $T$ acts by left multiplication: we choose a coset representative for $\delta$ with $x$-entry $t_{x}$ and $t_{1}=1$. Then we have, for each $y \in K=T, \delta=\delta^{y}$ and hence for each $x \in T, t_{x}=t_{y}^{-1} t_{y x}$ that is $t_{y} t_{x}=t_{y x}$. Thus there is an isomorphism $\sigma \in$ Aut $T$ such that $t_{x}=x^{\sigma}$. Now $L$ must act on the labels by right multiplication and we find that for $y \in L$, with $L$ identified with $T$, and for $\mathbf{x}=(x, \ldots, x) \in D, \mathbf{x} y$ fixes $\delta$ if and only if $\left(y z y^{-1}\right)^{\sigma x}=z^{\sigma}$ for all $z \in T$, and this holds if and only if $\sigma=y^{-1} \sigma x$, that is $y=\sigma x \sigma^{-1}$. Thus $\left(D \times\left(G_{\alpha} \cap P\right)\right)_{\delta}^{\Delta}=\left\{\left(\mathbf{x}, \sigma x \sigma^{-1}\right) \mid x \in T\right\}$.

Finally we consider the case where $G_{\alpha}$ is faithful and quasiprimitive on $\Delta$.

LEMMA 1.5. Suppose that $\Delta \neq\{\alpha\}$ is a faithful $G_{\alpha}$-orbit such that $D^{\Delta}$ is transitive.

(a) If $G_{\alpha} \cap P=1$ then $G_{\alpha} \simeq A(T)$ and $P \simeq G_{\alpha} /\left(G_{\alpha} \cap A(T)\right)$ which is isomorphic to a section of Out $T$ and hence is soluble.

(b) If $\left(G_{\alpha} \cap P\right)^{\Delta}$ is transitive then $G_{\alpha} \cap A(T)=D \simeq G_{\alpha} \cap P$; both $D$ and $G_{\alpha} \cap P$ are regular on $\Delta$. In this case $G_{\alpha}^{\Delta}$ is primitive. (If we identify $G_{\alpha} \cap P$ with $T$ then with $\delta=D\left(t_{1}, \ldots, t_{k}\right) \in \Delta$ and $t_{k}=1$, there is an automorphism $\sigma$ 
of $T$ such that $\left(D \times\left(G_{\alpha} \cap P\right)\right)_{\delta}=\left\{\left(\mathbf{x}^{\sigma}, x\right) \mid x \in T\right\}$ where $\mathbf{x}^{\sigma}=\left(x^{\sigma}, x^{\sigma}, \ldots, x^{\sigma}\right)$ and for all $1 \leq i \leq k$ we have $x^{-\sigma} t_{i} x^{\sigma}=t_{k x^{-1}}^{-1} t_{i x^{-1}}$.)

REMARKS 1.6. (a) In part (a) the stabilizer of $\delta$ in $D$ is $\left\{\mathbf{t} \mid t \in \bigcap_{1 \leq i \leq k} C_{T}\left(t_{i}\right)\right\}$ and as in Remark 1.4(c) we see that $G_{\alpha}^{\Delta}$ cannot be 2-transitive. In part (b), $G_{\alpha}^{\Delta}$ has two minimal normal subgroups and again cannot be 2-transitive. Thus we can conclude

COROLLARY 1.7. If $G$ has a simple diagonal action on $\Omega$ then $G$ has no 2-transitive subconstituents.

(b) In case (a) if we identify $D$ with $T$ then $D_{\delta}=\bigcap_{1 \leq i \leq k} C_{T}\left(t_{i}\right)$ that is $D_{\delta}$ is the centralizer in $T$ of the set $S(\delta)=\left\{t_{1}, t_{2}, \ldots, t_{k}=1\right\}$. Since $G_{\alpha}=D G_{\alpha \delta}$ we have $P=\Pi_{P}\left(G_{\alpha \delta}\right)$ and so for each $\tau \in P$ there is a $\sigma \in A(T)$ such that $\sigma \tau \in G_{\alpha \delta}$ : moreover $\sigma \tau \in G_{\alpha \delta}$ if and only if $t_{i}=\left(t_{k \tau^{-1}}^{-1} t_{i \tau^{-1}}\right)^{\sigma}$ for all $1 \leq i \leq k$. Now $\sigma \tau \in G_{\alpha}$ induces a map from $S(\delta)$ to $S\left(\delta^{\sigma \tau}\right)$ and $G_{\alpha \delta}$ fixes $S(\delta)$ setwise. If $G_{\alpha}^{\Delta}$ is primitive then $G_{\alpha \delta}$ must be the full setwise stabilizer of $S(\delta)$ in $G_{\alpha}$ (since the other possibility that $S(\delta)=S(\gamma)$ for all $\gamma \in \Delta$ is not allowed: for $S(\delta)$ would contain the conjugacy class in $D$ of each $t_{i}$ and hence $D_{\delta}=\bigcap C_{T}\left(t_{i}\right)=1$. This is not possible for an almost simple primitive group).

(c) In case (b) since $D$ is regular on $\delta$ we have

$$
\bigcap_{1 \leq i \leq k} C_{T}\left(t_{i}\right)=1 \text {. }
$$

Also since $G_{\alpha} \cap P$ is transitive on $\Delta$, each $\gamma=D\left(x_{1}, \ldots, x_{k}\right) \in \Delta$ with $x_{k}=1$ has its entries $x_{i}$ of the form $t_{j}^{-1} t_{l}$ for some $j, l$. It follows that the set $S$ of entries in points of $\Delta$ on the one hand is the union of the $T$-conjugacy classes of the $t_{i}$ (since $D^{\Delta}$ is transitive) and on the other hand is $\left\{t_{j}^{-1} t_{l} \mid j, l \in[1, k]\right\}$ : that is $S$ is a normal set with a kind of closure property. We make also the following observations.

(i) If $t \in G_{\alpha} \cap P=T$ fixes positions $k$ and $i$, for some $1 \leq i<k$ then $t^{\sigma}$ centralizes $t_{i}$.

(ii) If $\Delta$ is self-paired then $\delta^{-1}=D\left(t_{1}^{-1}, t_{2}^{-1}, \ldots, t_{k-1}^{-1}, 1\right) \in \Delta$ so, for some $\mathbf{t}=(t, \ldots, t) \in D, \delta^{\mathbf{t}}=\delta^{-1}$, that is $t$ inverts each $t_{i}$. Thus for any two such elements $t=x$ and $t=y$ say the product $x y^{-1}$ centralizes all the $t_{i}$ and we deduce $x y^{-1}=1$; so $t$ is unique and has order at most 2 .

ProOF OF LEMMA 1.5. (a) If $G_{\alpha} \cap P=1$ then $A(T)=\Pi_{A}\left(G_{\alpha}\right) \simeq G_{\alpha}$ and $P=\Pi_{P}\left(G_{\alpha}\right) \simeq G_{\alpha} /\left(G_{\alpha} \cap A(T)\right)$ which is isomorphic to a quotient of $A(T) / D$ : thus (a) holds.

(b) Suppose that $\left(G_{\alpha} \cap P\right)^{\Delta}$ is transitive. As in the proof of Lemma 1.3(c), $\left(G_{\alpha} \cap A(T)\right)^{\Delta}$ and $\left(G_{\alpha} \cap P\right)^{\Delta}$ centralise each other, so each is regular on $\Delta$ and 
$G_{\alpha} \cap A(T)=D \simeq G_{\alpha} \cap P$. Thus $G_{\alpha \delta} \cap\left(D \times\left(G_{\alpha} \cap P\right)\right)=\left\{\left(\mathbf{x}^{\sigma}, x\right) \mid x \in T\right\}$ for some $\sigma \in$ Aut $T$ where $\mathbf{x}^{\sigma}=\left(x^{\sigma}, x^{\sigma}, \ldots, x^{\sigma}\right)$ and so $x^{-\sigma} t_{i} x^{\sigma}=t_{k x^{-1}} t_{i x^{-1}}$ for all $i$.

\section{Primitive groups with a product action}

Here we assume that $G \leq X=H$ wr $S_{x}$ acts primitively on $\Omega=\Gamma^{x}=$ $\Gamma_{1} \times \cdots \times \Gamma_{x}$ for some $x \geq 2$ where the following hold.

(i) The group $H$ is a primitive permutation group on $\Gamma$ and $T \leq H \leq$ Aut $T$ for a nonabelian simple group $T$, or $H$ has a simple diagonal action on $\Gamma$ as described in the previous section, say $H$ has socle $T^{y}$ with $T$ a nonabelian simple group and $y \geq 2$.

(ii) The group induced by $G$ on $\Gamma_{i}$ is $H_{i}$, a copy of $H$ : the base group of $X$ is $H^{x}=H_{1} \times \cdots \times H_{x}$, and $G$ and $X$ have the same socle $N=T^{x y}=T_{1} \times \cdots \times T_{x y}$ (where $y=1$ if $H$ is almost simple and the socle of $H_{i}$ is

$$
\left.\operatorname{soc} H_{i}=T_{(i-1) y+1} \times \cdots \times T_{i y}\right) \text {. }
$$

(iii) The top group $S_{x}$ of $X$ permutes the sets $\mathcal{G}=\left\{\Gamma_{1}, \ldots, \Gamma_{x}\right\}$ and $\mathscr{H}=$ $\left\{H_{1}, \ldots, H_{x}\right\}$ naturally, and $G$ induces a transitive subgroup $P$ of $S_{x}$.

(iv) For $\alpha=(\gamma, \gamma, \ldots, \gamma) \in \Omega=\Gamma^{x}$ the stabilizer $G_{\alpha}=G \cap\left(H_{\gamma}\right.$ wr $\left.S_{x}\right), G_{\alpha}$ contains $N_{\alpha}=(\operatorname{soc} H)_{\gamma}^{x}$, and as $G=N G_{\alpha}, G_{\alpha}$ induces $P$ on $\mathcal{G}$ and $\not$.

THEOREM 2.1. Suppose that $G$ is a primitive permutation group on $\Omega$ with the product action as described above, and suppose that $\Delta$ is a $G_{\alpha}$-orbit in $\Omega \backslash\{\alpha\}$.

(a) If $G_{\alpha}$ is quasiprimitive on $\Delta$ then $\Delta=\Delta(\gamma)^{x}$ where $\Delta(\gamma)$ is an orbit of $H_{\gamma}$ in $\Gamma$.

(b) If $(H \mathrm{wr} P)_{\alpha}$ is quasiprimitive on $\Delta$ then $H_{\gamma}$ is quasiprimitive on $\Delta(\gamma)$.

(c) Also $(H \mathrm{wr} P)_{\alpha}$ is primitive on $\Delta$ if and only if $H_{\gamma}$ is primitive on $\Delta(\gamma)$. Thus if $G_{\alpha}$ is primitive on $\Delta$ then $H_{\gamma}$ is primitive on $\Delta(\gamma)$.

(d) The orbit $\Delta$ is self-paired for $G_{\alpha}$ if and only if $\Delta(\gamma)$ is a self-paired oribt of $H_{\gamma}$.

REMARKS 2.2. (a) We have not quite shown that the action of $H_{\gamma}$ on $\Delta(\gamma)$ must be quasiprimitive; the problem is that some $M \triangleleft H_{\gamma}$ with $M^{\Delta(\gamma)} \neq 1$ may be such that $\left(M^{x} \cap G_{\alpha}\right)^{\Delta}=1$, so that the quasiprimitivity of $G_{\alpha}^{\Delta}$ yields no information about the action of $M$ on $\Delta(\gamma)$. Can this situation really occur? (Note that $\left(M^{x} \cap G_{\alpha}\right)^{\Delta}=1$ implies that $\left(M \cap(\operatorname{soc} H)_{\gamma}\right)^{\Delta(\gamma)}=1$.) 
(b) If $\gamma^{\prime} \in \Delta(\gamma) \backslash\{\gamma\}$ then $\delta^{\prime}=\left(\gamma^{\prime}, \gamma^{\prime}, \gamma^{\prime}, \ldots, \gamma^{\prime}\right) \in \Delta$ and $\delta^{\prime \prime}=\left(\gamma^{\prime}, \gamma, \ldots, \gamma\right) \in$ $\Delta$ and it is not possible for an element of $G_{\alpha \delta}$ to map $\delta^{\prime}$ to $\delta^{\prime \prime}$. Thus

COROLLARY 2.3. A primitive group with product action has no 2-transitive subconstitutents.

In fact similar considerations show that $G_{\alpha}$ must have rank at least $x+1$ on $\Delta$ and that if $G_{\alpha}^{\Delta}$ has rank $x+1$ then $P \supseteq A_{x}$ and $H_{\gamma}^{\Delta(\gamma)}$ is 2-transitive.

PRoOF OF THEOREM 2.1. Let $\Delta_{0}(\gamma)=\{\gamma\}, \Delta_{1}(\gamma), \ldots, \Delta_{r}(\gamma)$ be the orbits of $H_{\gamma}$ in $\Gamma$ where $r \geq 1$. Let $S=\left\{\mathfrak{j}=\left(j_{1}, \ldots, j_{x}\right) \mid 0 \leq j_{i} \leq r\right.$ for each $i=1, \ldots, x\}$. Then $P$ acts on $S$ by permuting coordinates in the same way as it acts on the set $\mathcal{G}=\left\{\Gamma_{1}, \ldots, \Gamma_{x}\right\}$. Moreover the orbits of $H_{\gamma}$ wr $P$ in $\Omega$ are in one-to-one correspondence with the orbits of $P$ in $S$, namely the orbit $\Delta_{J}(\alpha)$ of $(H \text { wr } P)_{\alpha}$ in $\Omega$ corresponding to the orbit $J$ of $P$ in $S$ is the union of $\Delta_{\mathrm{j}}(\alpha)=\Delta_{j_{1}}(\gamma) \times \cdots \times \Delta_{j_{z}}(\gamma)$ over all $\mathbf{j} \in J$. Thus $\Delta \subseteq \Delta_{J}(\alpha)$ for some orbit $J \neq\{(0,0, \ldots, 0)\}$ of $P$ in $S$. By [10, Lemma 2.3] (soc $H)_{\gamma}$ acts nontrivially on $\Delta_{j}(\gamma)$ for all $j \neq 0$ and it follows that $(\operatorname{soc} H)_{\gamma}^{x}\left(\triangleleft G_{\alpha} \cap H^{x}\right)$ is nontrivial and $\frac{1}{2}$-transitive on $\Delta_{\mathrm{j}}(\alpha)$ for each $\mathrm{j} \in J$. Now $G_{\alpha}$ and $P$ induce the same action on $\mathcal{G}$ and hence on $S$ and hence $\Delta \cap \Delta_{\mathrm{j}}(\alpha) \neq \varnothing$ for each $\mathbf{j} \in J$. Thus ( $\operatorname{soc} H)_{\gamma}^{x}$ is nontrivial on $\Delta$, is normal in $G_{\alpha}$, and as $G_{\alpha}$ is quasiprimitive on $\Delta$ it follows that $(\operatorname{soc} H)_{\gamma}^{x}$ is transitive on $\Delta$. Then as $(\operatorname{soc} H)_{\gamma}^{x}$ fixes $\Delta \cap \Delta_{\mathfrak{\jmath}}(\alpha)$ setwise for each $\mathbf{j} \in J$ it follows that $J=\{\mathbf{j}=(j, j, \ldots, j)\}$ for some $j>0$, and so $\Delta_{J}(\alpha)=\Delta_{j}(\gamma)^{x}$.

Now $(\operatorname{soc} H)_{\gamma}^{x}$ is transitive on $\Delta$ and hence $\Delta=A_{1} \times \cdots \times A_{x}$ where each $A_{i}$ is an orbit of $(\operatorname{soc} H)_{\gamma}$ in $\Delta_{j}(\gamma)$. However by [10, Lemma 2.2(b)] the setwise stabilizer of $\Gamma_{i}$ in $G_{\alpha}$ induces $H_{\gamma}$ of $\Gamma_{i}$ and hence $A_{i}=\Delta_{j}(\gamma)$ for each $i$, that is, $\Delta=\Delta_{j}(\gamma)^{x}$. If $(H \operatorname{wr} P)_{\alpha}^{\Delta}$ is quasiprimitive then clearly $H_{\gamma}^{\Delta_{j}(\gamma)}$ is quasiprimitive, and if $(H \mathrm{wr} P)_{\alpha}^{\Delta}$ is primitive then also $H_{\gamma}^{\Delta_{j}(\gamma)}$ is primitive. Conversely if $H_{\gamma}$ is primitive on $\Delta_{j}(\gamma)$ then $(H \text { wr } P)_{\alpha}^{\Delta}$ is primitive by [3]. Finally $\Delta$ is clearly self paired if and only if $\Delta_{j}(\gamma)$ is self paired.

\section{Primitive groups with a doubly transitive subconstituent}

Suppose that $G$ is a primitive permutation group on $\Omega$ and that for $\alpha \in \Omega$, $G_{\alpha}$ is 2-transitive on one of its orbits $\Gamma(\alpha) \subseteq \Omega \backslash\{\alpha\}$. By Corollaries 1.7 and 2.3 $G$ has neither a simple diagonal action nor a product action on $\Omega$. It follows from the O'Nan Scott Theorem [1] that Theorem A is true. In this section we shall discuss the case where $G$ has a unique minimal normal subgroup $N$ which is regular on $\Omega$. We consider first the case where $N$ is abelian. 
Proposition 3.1. Suppose that $G$ is a simply transitive primitive permutation group on $\Omega$ with an elementary abelian regular normal subgroup $N$, and suppose that $G_{\alpha}, \alpha \in \Omega$, is doubly transitive on one of its orbits $\Gamma(\alpha) \subseteq \Omega \backslash\{\alpha\}$. We identify $\Omega$ with $N$ in such a way that $\alpha$ is identified with the zero of $N$ (written additively).

(a) Then the orbit $\Gamma^{*}(\alpha)$ paired with $\Gamma(\alpha)$ is $-\Gamma(\alpha)=\{-\beta \mid \beta \in \Gamma(\alpha)\}$.

(b) The orbit $\Gamma(\alpha)$ is self-paired if and only if $N$ is a 2-group.

(c) The orbit $\Gamma \circ \Gamma^{*}(\alpha)=\{\gamma \mid$ there is a $\beta$ such that $\beta \in \Gamma(\alpha) \cap \Gamma(\gamma)$, and $\gamma \neq \alpha\}$ is self-paired.

Proof. Identifying $\Omega$ with $N$ such that $\alpha=0$, for each $\beta \in \Gamma(\alpha)$ we can translate by $-\beta \in N$ to obtain $-\beta \in \Gamma^{*}(\alpha)$. Hence $\Gamma^{*}(\alpha)=-\Gamma(\alpha)=\{-\beta \mid \beta \in$ $\Gamma(\alpha)$ \}. Thus if $N$ is a 2-group then $\beta=-\beta$ and so $\Gamma(\alpha)$ is self-paired. Conversely if $\Gamma(\alpha)$ is self-paired then $\{\beta,-\beta\}$ is a block of imprimitivity in $\Gamma(\alpha)$ for $G_{\alpha}$ and so $\beta=-\beta$, that is $N$ is a 2 -group.

Finally if $\beta$ and $\gamma$ are distinct points of $\Gamma(\alpha)$ then translating $(\alpha,-\beta)$ and $(\alpha,-\gamma)$ by $\gamma \in N$ and $\beta \in N$ respectively we find that $\gamma-\beta \in \Gamma^{*}(\gamma) \backslash\{\alpha\} \subseteq$ $\Gamma \circ \Gamma^{*}(\alpha)$ and $\beta-\gamma \in \Gamma^{*}(\beta) \backslash\{\alpha\} \subseteq \Gamma \circ \Gamma^{*}(\alpha)$. It follows as above that $\Gamma \circ \Gamma^{*}(\alpha)$ is self-paired. ( $\Gamma \circ \Gamma^{*}(\alpha)$ was shown to be a $G_{\alpha}$-orbit in [2].)

REMARKS 3.2. (a) A similar argument shows that for $\beta \in \Gamma(\alpha)$ we have $i \beta$ in $\Gamma(\alpha)$ where $i \in \mathbf{Z}$, if and only if $i \beta=\beta$ (since the set of $i \beta$ in $\Gamma(\alpha)$, $i \in \mathbf{Z}$, is a block of imprimitivity for $G_{\alpha}$ in $\Gamma(\alpha)$ ). In particular if $N=\mathbf{Z}_{p}^{d}$ and we regard $G_{\alpha}$ as a subgroup of $G L(d, p)$ then $G_{\alpha}$ contains no nontrivial scalar transformations. Similarly if in fact $G_{\alpha} \leq \Gamma L\left(d / a, p^{a}\right)$ then $G_{\alpha}$ contains no nontrivial $G F\left(p^{a}\right)$-scalar transformations.

(b) The stabilizer $G_{\alpha}$ regarded as a subgroup of $G L(d, p)$ is irreducible since $G$ is primitive, and so we could choose a basis so that $\Gamma(\alpha)$ contains all the standard basis vectors $e_{i}=\left(o^{i-1} 10^{d-i}\right), 1 \leq i \leq d$. Suppose that $|\Gamma(\alpha)|=d$ : then $G_{\alpha}$ permutes the standard basis vectors amongst themselves and so fixes the point $e_{1}+e_{2}+\cdots+e_{d} \neq \alpha$. This contradicts the fact that $G$ is primitive, so always $|\Gamma(\alpha)|>d$.

Now we consider the case where $G$ has a unique minimal normal subgroup $N$ which is nonabelian and regular on $\Omega$. Here (see [1] and [7]) $N=T_{1} \times \cdots \times T_{k}$ where each $T_{i}$ is isomorphic to a fixed nonabelian simple group $T$ and $k \geq 2$ : the group $G$ is a twisted wreath product $T \operatorname{twr}_{\varphi} P$, where $P$ is a transitive subgroup of $S_{k}$ permuting the $T_{i}$ naturally, and the twisting homomorphism $\varphi: P_{1} \rightarrow$ Aut $T$ is such that $\varphi\left(P_{1}\right)$ contains the group of inner automorphisms of $T$. We can identify $\Omega$ with $N$ so that $\alpha$ is identified with the identity $(1,1, \ldots, 1)$ of $N$, and then $G_{\alpha}=P$. 
Proposition 3.3. Suppose that $G=T \operatorname{twr}_{\varphi} P$ is primitive on $\Omega$ with regular normal subgroup $N=T^{k}$ as described above. Suppose that $G_{\alpha}$ is doubly transitive on an orbit $\Gamma(\alpha) \subseteq \Omega \backslash\{\alpha\}$, where $\Omega$ is identified with $N$ and $\alpha=(1,1, \ldots, 1)$. Then $G_{\alpha}$ has an orbit $\hat{\Gamma}(\alpha)$ in $\Omega \backslash\{\alpha\}$, possibly equal to $\Gamma(\alpha)$, such that the actions of $G_{\alpha}$ on $\Gamma(\alpha)$ and $\hat{\Gamma}(\alpha)$ are permutationally equivalent, and for $\beta=$ $\left(\beta_{1}, \ldots, \beta_{k}\right) \in \hat{\Gamma}(\alpha)$ all the nontrivial $\beta_{i}$ lie in a single $\varphi\left(P_{1}\right)$-conjugacy class $C$ and $G_{\alpha \beta}(\leq P)$ is transitive on the support $\Delta(\beta)=\left\{i \mid \beta_{i} \neq 1\right\}$ of $\beta$.

If $\Gamma(\alpha)$ is self-paired then $\hat{\Gamma}(\alpha)$ is also self-paired and $\beta$ has order 2 as an element of $N$.

Proof. Choose a transversal $\left\{\rho_{1}=1, \rho_{2}, \ldots, \rho_{k}\right\}$ for $P_{1}$ in $P$ such that $1 \rho_{i}=i$ for all $i$. Then each $\sigma \in G_{\alpha}=P$ is such that the $j$ th entry of $\beta^{\sigma}$ is

$$
\beta_{i}^{\rho_{i} \sigma \rho_{j}^{-1}}
$$

where $i=j \sigma^{-1}$ and we write $t^{\tau}$ for the image of $t \in T$ under $\tau \in P$. Let $\Delta_{1}, \ldots, \Delta_{r}$ be the $G_{\alpha \beta}$-orbits in $\mathbf{k}=\{1,2, \ldots, k\}$; we note that all $\beta$-entries in a fixed $\Delta_{i}$ are conjugate under $\varphi\left(P_{1}\right)$. Call $\Delta_{i}$ nontrivial if the entries in $\Delta_{i}$-positions in $\beta$ are not the identity. As $\beta \neq \alpha$, there is at least one nontrivial orbit, say $\Delta$. Define $\beta(\Delta) \in N$ to have entries equal to $\beta$-entries at positions in $\Delta$, and entries equal to 1 otherwise. Then $G_{\alpha \beta}$ fixes $\beta(\Delta)$, and as $G_{\alpha \beta}$ is a maximal subgroup of $G_{\alpha}$ and $G_{\alpha}$ does not fix the point $\beta(\Delta) \neq \alpha$, it follows that $G_{\alpha \beta}=G_{\alpha \beta(\Delta)}$. If $\hat{\Gamma}(\alpha)$ is the $G_{\alpha^{-}}$orbit containing $\beta(\Delta)$ then the $G_{\alpha^{-}}$ actions on $\Gamma(\alpha)$ and $\hat{\Gamma}(\alpha)$ are equivalent and $G_{\alpha \beta(\Delta)}$ is transitive on $\Delta$. Finally $\beta^{-1} \in \Gamma^{*}(\alpha)$ and arguing as in Proposition 3.1 we see that $\Gamma(\alpha)$ is self-paired if and only if $\beta=\beta^{-1}$ that is if and only if $\beta$ has order 2 in $N$. In this case $\beta(\Delta)$ also has order 2 so that $\hat{\Gamma}(\alpha)$ is also self-paired.

3.4. Further discussion of the twisted wreath product case. Let us assume that $\beta=\beta(\Delta)$ (that is replace $\Gamma(\alpha)$ by $\hat{\Gamma}(\alpha)), G_{\alpha \beta}$ is transitive on $\Delta(\beta)$, and all $\beta$ entries in $\Delta(\beta)$-positions lie in the $\varphi\left(P_{1}\right)$-conjugacy class $C$. We define a design $D$ as follows: the set of points is $\Gamma(\alpha)$, the set of blocks is $\mathbf{k}=\{1,2, \ldots, k\}$ with $\beta$ incident with $i$ whenever $i \in \Delta(\beta)$, that is $\beta_{i} \neq 1$. Then $G_{\alpha}$ acts faithfully (since $N$ is regular) as an automorphism group of this design; $G_{\alpha}$ is 2-transitive on points, transitive on blocks, and the stabilizer of a point is transitive on the blocks incident with the point. A counting argument shows that each pair of points is incident with

$$
\lambda=l(v l-k) / k(v-1)
$$

blocks where $l=|\Delta(\beta)|$. Of course $D$ is a degenerate design when $l=k$ but if $l<k$ then $k \geq v$. We note that the parameter $\lambda$ is $|\Delta(\beta) \cap \Delta(\gamma)|$ for distinct $\beta, \gamma$ in $\Gamma(\alpha)$. 
Further if $\beta, \gamma$ are distinct points of $\Gamma(\alpha)$ then the points $\beta^{-1} \gamma$ and $\gamma^{-1} \beta$ lie in $\Gamma \circ \Gamma^{*}(\alpha)$. Thus points of $\Gamma \circ \Gamma^{*}(\alpha)$ have $l_{2}=k-2 l+\lambda+L$ entries equal to 1 since there are $k=2 l+\lambda$ entries $i$ with $\beta_{i}=\gamma_{i}=1$ and $L \geq 0$ entries $i$ with $\beta_{i}=\gamma_{i} \neq 1$. The parameters $L, l_{2}$ can be determined in terms of $v, l, k$ and $|D|$ as follows:

(i) Given $i \in \mathbf{k}$ and $t \in \mathcal{C}$ the number $n(t, i)$ of $\beta \in \Gamma(\alpha)$ with $\beta_{i}=t$ is independent of $i$ and $t$.

PROOF. The subset of $P$ consisting of those elements which map $i$ to 1 is $\rho_{i}^{-1} P_{1}$; for each $\sigma \in \rho_{i}^{-1} P_{1}$ and each $\beta \in \Gamma(\alpha)$ with $\beta_{i}=t$ we have

$$
\left(\beta^{\sigma}\right)_{1}=\beta_{i}^{\rho_{i} \sigma}=t^{\rho_{i} \sigma}
$$

Thus $n(t, i)=n\left(t^{\rho_{i} \sigma}, 1\right)$ for all $\rho_{i} \sigma \in P_{1}$, that is $n(t, i)=n\left(t^{\prime}, 1\right)$ for all $t^{\prime} \in C$.

(ii) Counting the nonidentity entries in points of $\Gamma(\alpha)$ we obtain $v l=k n|C|$, where $n=n(t, i)$ above.

(iii) Counting triples $(\beta, \gamma, i)$, where $\beta, \gamma$ are in $\Gamma(\alpha)$ and the entry $i$ is such that $\beta_{i}=\gamma_{i} \neq 1$ we obtain $v(v-1) L=k|C| n(n-1)$, and subsituting for $n$

$$
L=(v l-k|C|) l / k|C|(v-1)
$$

One final remark about the self-paired case (where $\beta^{2}=1$ ): by a theorem of Baer and Suzuki, (see $[6,3.8 .2]$ ), there is some entry in $\beta \gamma$ whose order as an element of $T$ is not a power of 2. In particular $\beta \gamma \neq \gamma \beta$, that is no pair of distinct elements of $\Gamma(\alpha)$ commutes. Further if $\beta \gamma$ had odd order then the elements of $C$ would be isolated in any Sylow 2-subgroup of $T$ which would give a contradiction by Glauberman's $Z^{*}$-theorem [5]. Thus the order of $\beta \gamma$ is an even integer, not a power of 2.

Now $\beta \gamma \beta$ has order 2 (as it is conjugate in $N$ to $\gamma$ ) and is joined to $\gamma \beta$ so it lies in $\Gamma(\alpha) \cup \Gamma_{3}(\alpha)$ where $\Gamma_{3}(\alpha)=\Gamma \circ \Gamma \circ \Gamma(\alpha) \backslash \Gamma(\alpha)$. If $\beta \gamma \beta \in \Gamma(\alpha)$, then as it is joined to $(\gamma \beta)^{2}$, and $(\gamma \beta)^{2}$ does not have order 2 we would have $(\gamma \beta)^{2} \in \Gamma_{2}(\alpha)$. Then $\gamma \beta$ and $(\gamma \beta)^{2}$ would have the same order, and hence would have odd order, a contradiction. Thus $\beta \gamma \beta \in \Gamma_{3}(\alpha)$. If $G_{\alpha}$ were transitive on $\Gamma_{3}(\alpha)$ also, then all entries in points of $\Gamma_{3}(\alpha)$ would lie in $C \cup\{1\}$. It follows that $T=\{1\} \cup \mathcal{C} \cup \mathcal{C}^{2}$. This seems to be a strong restriction on the group $T$, and hence on $\Gamma$.

3.5. Final Remarks. Suppose that $G$ is primitive on $\Omega$ and that $G_{\alpha}$ is 2-transitive and unfaithful on an orbit $\Gamma(\alpha) \subseteq \Omega \backslash\{\alpha\}$. Then it follows from Theorem A that $T \leq G \leq$ Aut $T$ for some nonabelian simple group $T$. Can such groups be classified? 


\section{References}

[1] M Aschbacher and L. L. Scott, 'Maximal subgroups of finite groups', J. Algebra 92 (1985), 44-80.

[2] P. J. Cameron, 'Permutation groups with multiply transitive suborbits I', Proc. London Math. Soc. (3) 25 (1972), 427-440; II Bull. London Math. Soc. 6 (1974), 1-5.

[3] P. J. Cameron, 'Finite permutation groups and finite simple groups', Bull. London Math. Soc. 13 (1981), 1-22.

[4] P. J. Cameron and C. E. Praeger, 'Graphs and permutation groups with projective subconstitutents', J. London Math. Soc. (2) 25 (1982), 62-74.

[5] G. Glauberman, 'Central elements in core-free groups', J. Algebra 4 (1966), 403-420.

[6] D. Gorenstein, Finite groups (Harper and Row, New York, Evanston and London, 1968).

[7] L. G. Kovacs, 'Maximal subgroups in composite groups', J. Algebra, 99 (1986), 114-131.

[8] C. E. Praeger, 'On primitive permutation groups with a doubly transitive suborbit', $J$. London Math. Soc. (2) 17 (1978), 67-73.

[9] C. E. Praeger, 'Primitive permutation groups and a characterization of the odd graphs', J. Combin. Theory Ser. B 31 (1981), 117-142.

[10] C. E. Praeger, J. Saxl, and K. Yokoyama, 'Distance transitive graphs and finite simple groups', Proc. London Math. Soc. (3) 55 (1987), 1-21.

[11] L. L. Scott, 'Representations in characteristic p', Proc. Sympos. Pure Math. 37 (1980), 319-331.

[12] H. Wielandt, Finite permutation groups (Academic Press, New York, 1964).

\section{Department of Mathematics}

The University of Western Australia

Nedlands, Western Australia 6009

Australia 